\title{
Dynamics of attentional and oculomotor orienting in visual foraging tasks
}

\begin{tabular}{|c|c|}
\hline Journal: & Quarterly Journal of Experimental Psychology \\
\hline Manuscript ID & QJE-SIP-19-280.R1 \\
\hline Manuscript Type: & Special Issue Paper \\
\hline $\begin{array}{r}\text { Date Submitted by the } \\
\text { Author: }\end{array}$ & 09-Dec-2019 \\
\hline Complete List of Authors: & $\begin{array}{l}\text { Tagu, Jérôme; University of Iceland School of Health Sciences, Icelandic } \\
\text { Vision Lab } \\
\text { Kristjánsson, Árni; University of Iceland School of Health Sciences, } \\
\text { Icelandic Vision Lab; National Research University Higher School of } \\
\text { Economics, School of Psychology }\end{array}$ \\
\hline Keywords: & $\begin{array}{l}\text { Visual foraging, Visual search, Visual attention, Eye movements, Search } \\
\text { strategy }\end{array}$ \\
\hline
\end{tabular}

\section{SCHOLARONE" \\ Manuscripts}


Runing head: Oculomotor dynamics in visual foraging

\title{
Dynamics of attentional and oculomotor orienting in visual foraging tasks
}

\author{
Jérôme Tagu ${ }^{1} \&$ Árni Kristjánsson ${ }^{1,2}$ \\ ${ }^{1}$ Icelandic Vision Laboratory, Faculty of Psychology, School of Health Sciences, University of \\ Iceland, Reykjavík, Iceland \\ 2 School of Psychology, National Research University Higher School of Economics, Moscow, \\ Russian Federation
}

Correspondence should be addressed to:

Jérôme Tagu, tagu@hi.is

Icelandic Vision Laboratory, Faculty of Psychology, University of Iceland

Nýi Garður, Sæmundargötu 12, 101 Reykjavík, Iceland

Manuscript information:

- Title: 73 characters

- Running title: 38 characters

- Abstract: 194 words

- Text: 8851 words

- 6 Figures, 1 Table 
Runing head: Oculomotor dynamics in visual foraging

\begin{abstract}
A vast amount of research has been carried out to understand how humans visually search for targets in their environment. However, this research has typically involved search for one unique target among several distractors. Although this line of research has yielded important insights into the basic characteristics of how humans explore their visual environment, this may not be a very realistic model for everyday visual orientation. Recently, researchers have used multi-target displays to assess orienting in the visual field. Eye movements in such tasks are however less well understood. Here we investigated oculomotor dynamics during four visual foraging tasks differing in target crypticity (feature-based vs. conjunction-based foraging) and the effector type being used for target selection (mouse-foraging vs. gazeforaging). Our results show that both target crypticity and effector type affect foraging strategies. These changes are reflected in oculomotor dynamics, feature-foraging being associated with focal exploration (long fixations and short-amplitude saccades) and conjunction-foraging with ambient exploration (short fixations and high-amplitude saccades). These results provide important new information for existing accounts of visual attention and oculomotor control, and emphasize the usefulness of foraging tasks for a better understanding of how humans orient in the visual environment.
\end{abstract}

Keywords: Visual foraging, Visual search, Visual attention, Eye movements, Search strategy 
Runing head: Oculomotor dynamics in visual foraging

\section{Introduction}

Visual search is a commonly used task for measuring visual attention (see Kristjánsson, 2015 and Wolfe \& Horowitz, 2017, for recent reviews). A large part of the visual search literature is based on behavioural results, where manual reaction time and accuracy are commonly used to measure attentional orienting (e.g., Moran, Zehetleitner, Liesefeld, Müller, \& Usher, 2016). In covert visual search tasks, a distinction is typically made between parallel processing of items composed of individual features and serial processing of complex items, involving the binding of several features (Feature Integration Theory, Treisman \& Gelade, 1980). Overt visual search, where participants make eye movements to find the target in the display, has been considered to proceed serially, because only one saccade is executed at a

time. Hence, models of eye movement programming propose that each saccade is programmed separately during the preceding fixation (e.g., Findlay \& Walker, 1999). This research has however often involved sparse displays composed of a single target and a few distractors, where only one saccade per trial is executed (for reviews, see Eckstein, 2011; Rayner, 2009). In more complex displays requiring the execution of several saccades to identify the target, the dissociation between serial and parallel search instead concerns the time at which the second or third saccades are planned. Does the oculomotor system plan the sequence of saccades in advance, before the execution of the first saccade, or does it plan one saccade at a time in a serial manner, just before their execution? The first evidence for parallel programming of sequences of saccades comes from the double-step paradigm (McLaughlin, 1967), in which two saccadic targets are presented when the eyes are still at fixation, and where participants are asked to make two successive saccades to these targets. Becker \& Jürgens (1979) showed that secondary saccades in such paradigms can have very short latencies of less than $100 \mathrm{~ms}$, so that little or no visual processing can take place in such short fixation periods. The authors therefore proposed that these saccades must have been planned in advance, before the execution of the preceding saccade. 
Runing head: Oculomotor dynamics in visual foraging

\section{From sequences of saccades to scanpaths}

A large literature has subsequently emerged on the temporal and spatial dynamics of sequences of saccades, providing insights into the serial or parallel planning of eye movements during visual search (for a review, see Liversedge \& Findlay, 2000). Zelinsky, Rao, Hayhoe, \& Ballard (1997) interestingly reported a dissociation between manual and oculomotor data obtained from visual search tasks, showing that even though manual reaction times increased with stimulus set size, favouring the serial processing hypothesis; analysis of eye movements during the task suggested parallel processing of visual stimuli. The authors showed that the first saccades did not land on the target position, but rather on the "centre of gravity" of the visual scene (global effect; Coren \& Hoenig, 1972; Findlay, 1982; He \& Kowler, 1989). They argued that this behaviour reflected parallel processing of the entire display, not just the target. Subsequently, it was only the second or the third saccade that reached the target, suggesting that the search evolves from a global/parallel mode to a local/serial mode during the eye movement sequence.

Noton \& Stark (1971) were the first to propose that the sequence of eye movements leading to target selection during visual search tasks could provide important information about the dynamics of visual attention. They proposed a "scanpath theory", claiming that the sequence of eye movements generated to a given picture would be the same both within and between individuals. Although this theory has raised great interest and inspired many studies, it has subsequently been criticized. Groner, Walder, \& Groner (1984) showed for example that individuals could explore faces using either "global" or "local" scanpaths. Global scanpaths are composed of numerous high-amplitude saccades, whereas local scanpaths are mostly composed of short-amplitude saccades (see also Zangemeister, Sherman, \& Stark, 1995). Unema, Pannasch, Joos, \& Velichkovsky (2005) then modelled oculomotor behaviour during visual scene exploration by examining the relationship between the duration of a given fixation and the amplitude of the subsequent saccade. They proposed that exploration in an ambient mode is characterized by short-duration fixations followed by high-amplitude 
Runing head: Oculomotor dynamics in visual foraging

saccades, whereas exploration in a focal mode is characterized by long-duration fixations followed by short-amplitude saccades. The ambient mode is considered to be related to bottom-up processing in the dorsal visual stream, whereas the focal mode is thought to be related to top-down processing in the ventral visual stream. Some authors have subsequently suggested that indices for the visual exploration mode being used can be calculated from the relationship between fixation duration and saccade amplitude (Goldberg \& Kotval, 1999; Krejtz, Duchowski, Krejtz, Szarkowska, \& Kopacz, 2016; Velichkovsky, Rothert, Kopf, Dornhöfer, \& Joos, 2002). Over, Hooge, Vlaskamp, \& Erkelens (2007) have shown that during visual search tasks, exploration gradually evolves from an ambient to a focal mode.

\section{Visual foraging}

So far, investigations of eye movement properties during visual search tasks have yielded important insights into how humans explore the visual world to find the object of interest. In everyday life however, the visual world is rarely so simple as to involve only one target, and we often search for several targets simultaneously, or for several instances of several target types simultaneously, among several instances of several distractor types. This is for example the case in a football match, where there are many targets (players on your team) and distractors (the members of the other team and the referee), or when we simultaneously look for several items from a shopping list in a supermarket while avoiding many other products on the shelves. Obviously, foraging behaviours in such complex visual environments are not captured by standard visual search tasks where one unique target is presented among a few distractors.

Multi-target foraging tasks have mainly been examined in animal research (e.g., Dukas, 2002; Dukas \& Ellner, 1993; Tinbergen, 1960). When foraging for food and when food sources are conspicuous, predators frequently switch between different prey types; but when prey are hard to find, predators change their strategy and focus on a single prey type and 
Runing head: Oculomotor dynamics in visual foraging

exhaust the entire category before switching to another prey type (Dawkins, 1970; Dukas, 2002; Dukas \& Ellner, 1993; Tinbergen, 1960). A line of research on human visual foraging has subsequently emerged (Cain \& Mitroff, 2013; Hills, Kalff \& Wiener, 2013; Kristjánsson, Jóhannesson, \& Thornton, 2014; Kristjánsson \& Kristjánsson, 2018; Kristjánsson, Thornton, \& Kristjánsson, 2018; Wolfe, 2013). Kristjánsson et al. (2014) addressed whether humans foraged in the same way as animals, adjusting their foraging strategies depending on target crypticity. Human participants performed a foraging task on an iPad. Their task was to select forty targets from two different categories by tapping on them with their forefinger, without selecting any of the forty distractors. In a feature-based foraging task, targets were conspicuous (e.g. red and green dots among yellow and blue distractor dots), whereas in a conjunction-based foraging task, targets were more cryptic (e.g. red dots and green squares among green dots and red squares). The main findings were that humans did indeed forage like animals do and adapted their strategy to target crypticity. They frequently switched between the two target types during feature foraging, but had difficulties doing so during more cryptic conjunction foraging. Instead, they focused on a single target type (e.g., red dots) until the entire category was exhausted before turning to the second target type (e.g., green squares). Importantly however, in Kristjánsson et al. (2014) a subset of participants did not change their strategy and continued to frequently switch between target types during conjunction foraging. The authors called these individuals "super-foragers". Follow-up studies have shown that although these individual differences in foraging behaviour are linked to cognitive abilities in children (Ólafsdóttir, Gestsdóttir, \& Kristjánsson, 2019; Ólafsdóttir, Kristjánsson, Gestsdóttir, Jóhannesson, \& Kristjánsson, 2016), such correlations have not been observed for adults (Jóhannesson, Kristjánsson, \& Thornton, 2017), suggesting that other factors are involved. In the end, although these findings revealed that animal and human foraging are driven by similar mechanisms and proceed in a similar way, the individual differences arising in humans' behaviour are still poorly understood, highlighting that many mechanisms guiding visual foraging are still unknown. As has been the case for single-target visual search, we propose that investigating eye movement dynamics during 
Runing head: Oculomotor dynamics in visual foraging

visual foraging tasks may provide important additional information regarding foraging strategies, and more generally regarding visual orienting in the visual field. While exploring a visual scene, each individual exhibits specific oculomotor dynamics that vary between observers but are stable for individuals (Bargary et al., 2017; Foulsham, Frost, \& Sage, 2018; Tagu, Doré-Mazars, Vergne, Lemoine-Lardennois, \& Vergilino-Perez, 2018a, 2018b). Differences that have previously been found between "normal-" and "super-" foragers during finger foraging may therefore be reflected in individual differences in oculomotor behaviour. We therefore expect feature- and conjunction foraging to be associated with different eye movement dynamics. We notably expect individuals to explore the scene with a bottom-up ambient mode of visual exploration during feature foraging, while during conjunction foraging we expect them to explore the scene with a more top-down focal mode of visual exploration. Furthermore, these dynamics should vary according to individuals' foraging strategy, i.e., between normal and super foragers.

Jóhannesson, Thornton, Smith, Chetverikov, \& Kristjánsson (2016) investigated gaze foraging, where participants had to select the targets with their eyes instead of their fingers. The same participants also performed the finger foraging task from Kristjánsson et al. (2014). Note however that eye movement dynamics were not assessed during finger foraging. During this task, the authors replicated the results of Kristjánsson et al. (2014), reinforcing the idea that foraging behaviour is similar between animals and humans. Importantly, this study again revealed a subset of super-foragers who continued to frequently switch between the two target categories during conjunction foraging. During gaze foraging, however, participants mostly switched frequently between the two target categories, irrespective of target crypticity (i.e., most of them foraged like super-foragers when foraging with eye gaze). However, several methodological factors could have led to the observed differences between finger- and gaze foraging. For example, trials contained 80 stimuli during finger foraging against only 32 for gaze foraging. The fewer stimuli in the display may have rendered the task easier, and although these initial results suggest that different mechanisms may be 
Runing head: Oculomotor dynamics in visual foraging

involved when we use fingers and eye-gaze to forage, drawing clear conclusions was difficult since the methodological differences complicated any comparisons. In the current study, we examined eye movement dynamics during multi-target foraging more thoroughly, where observers foraged both using a computer mouse and eye gaze. Eye movements were recorded in both tasks, and we displayed an equal number of stimuli in both the gazeforaging and the mouse-foraging tasks, so that the visual appearance of the stimuli in both cases was identical, allowing full comparison between tasks. During mouse-foraging, we expected to replicate the findings of Kristjánsson et al. (2014) with frequent switches between target categories during feature foraging and few switches during conjunction foraging. Based on the study of Jóhannesson et al. (2016), this dynamic would probably be modified when foraging with eye gaze, where we expected smaller differences between feature- and conjunction foraging.

\section{Methods}

\section{Participants}

24 individuals (21 naïve undergraduate students, 2 naïve graduate students, and 1 nonnaïve member of the laboratory) participated in this study. They were aged from 20 to 29 years-old (mean age $=24$, s.d. $=2.5$ ) and included 18 females. All participants were right handed (Edinburgh Handedness Inventory, Oldfield, 1971, mean laterality score $=77 \%$, s.d. $=20 \%$ ) and 16 of them were right-eye-dominant (hole-in-card test, Durand \& Gould, 1910). The undergraduate students received course credits in exchange for their participation, while the two graduate students and the lab member participated without compensation. Prior to their inclusion in the study, participants received clear explanations about the procedure and gave their written informed consent. The study was completed in accordance with the requirements of the ethics committee at the University of Iceland and conformed with the ethical guidelines set out by the 1964 declaration of Helsinki and its later amendments. 
Runing head: Oculomotor dynamics in visual foraging

\section{Instruments and materials}

Stimuli were presented on a BenQ XL2411Z monitor (BenQ, Taipei, Taiwan) with a refresh rate of $144 \mathrm{~Hz}$ and a resolution of $1920 \times 1080$ pixels. The experiment took place in a dimly lit and soundproof room. Participants were seated $57 \mathrm{~cm}$ away from the monitor and their heads were kept stable with a chin and forehead rest. In all tasks, eye movements were binocularly recorded using an EyeLink 1000 Plus (SR Research, Ontario, Canada) sampled at $1000 \mathrm{~Hz}$ and with an average spatial accuracy of $0.15^{\circ}$. The online saccade detection corresponded to an above-threshold velocity $(30 \% \mathrm{~s})$ and acceleration $\left(>8000 \% \mathrm{~s}^{2}\right)$.

Each trial involved eighty stimuli (40 targets, 40 distractors) equalized in size $\left(0.5^{\circ}\right.$ diameter $)$ and luminance $\left(14 \mathrm{~cd} / \mathrm{m}^{2}\right)$ that were presented on a dark grey background with a luminance of $7 \mathrm{~cd} / \mathrm{m}^{2}$. As shown in Figure 1 , stimuli were randomly distributed across a non-visible $10 \times 8$ grid occupying $24^{\circ} \times 19^{\circ}$ of the visual field. The rows/columns of the grid were separated by an empty space of about $2.5^{\circ}$. The position of the stimuli within the grid was however slightly jittered $\left( \pm 0.48^{\circ}\right)$ to create a less uniform appearance, and the initial $2.5^{\circ}$ inter-stimuli distance changed accordingly. The overall spatial layout and location of targets and distractors was generated independently on every trial.

\section{Procedure}

Participants had to perform four foraging tasks, differing in target crypticity (feature-based or conjunction-based foraging) and by the effector-type used to select the targets (computer mouse or eye gaze). Examples of feature-foraging and conjunction-foraging displays are presented in Figure 1a and 1b, respectively. All tasks were composed of two training trials and sixteen test trials, and were all completed in one single session of about $1 \mathrm{~h} 30 \mathrm{~min}$. 
Runing head: Oculomotor dynamics in visual foraging

Insert Figure 1 about here

There were two target types and two distractor types, differing by their colour in the feature foraging tasks (i.e., red dot and green dot targets among yellow dot and blue dot distractors, or the reverse) or by the combination of their colour and shape in the conjunction foraging tasks (i.e., red square and green dot targets among red dot and green square distractors, or the reverse). On each trial, participants were instructed to select all the targets in the display as fast as possible, without selecting any distractor. When a target was selected, it disappeared, whereas distractor selection led to an error-message screen and to the renewal of the trial until successful completion. In other words, if participants selected a distractor, the entire foraging array with the eighty stimuli was presented again (with new randomly assigned stimulus locations), until all forty targets had been successfully selected. When the trial was completed, a feedback screen appeared, indicating the progression in the experiment and the trial response time.

In the mouse foraging tasks, participants were asked to select the targets by clicking on them with the left button of a computer mouse. In the gaze foraging tasks, they had to do so by fixating the targets with their eyes. In both tasks, the stimuli were surrounded by a $1.5^{\circ}$ interest area, and the stimulus selection was triggered when a mouse click or an eye fixation was detected in that area. The inter-target distance and spatial jitter applied to stimuli locations were chosen so that the interest areas never overlapped. During gaze foraging, target selection was triggered when an eye fixation from the dominant eye lasting longer than $200 \mathrm{~ms}$ was detected in the interest area. To avoid erroneous selection of the distractors when the participants were exploring the visual field in search for other targets, the fixation duration needed for distractor selection (and for displaying the error message) was increased to $350 \mathrm{~ms}$. These fixation times were chosen based on pre-tests run on two well-trained 
Runing head: Oculomotor dynamics in visual foraging

participants, where $200 \mathrm{~ms}$ was the optimal timing to prevent from false detections of target selections during visual exploration without affecting the distribution of fixation durations, and $350 \mathrm{~ms}$ was the optimal timing to prevent omissions of distractor selections while allowing individuals to quickly identify the stimuli as distractors and continue exploring the scene (especially at the end of the trials, when only one target remained in the display, together with the forty distractors).

The order of the tasks was counterbalanced so that half of the participants started with the mouse foraging tasks, while half performed feature foraging before conjunction foraging. The target identities were counterbalanced as well, so that during feature foraging, half of the participants saw red and green targets among yellow and blue distractors while others saw the reverse; and during conjunction foraging, half of the participants saw red square and green dot targets among red dot and green square distractors while others saw the reverse. For a given observer, target and distractor identities were held constant between mouse and gaze foraging tasks.

\section{Data analysis}

In line with previous studies (Jóhannesson et al., 2016; Kristjánsson et al., 2014), our primary behavioural dependent variable was the number of "runs" on a given trial. A "run" refers to the sequential selection of targets of the same category. As such, with 40 targets divided into two categories, the number of runs could vary between two and 40 . The number of runs is inversely related to run length (i.e., the number of elements selected in a run, ranging from one to 20), so that constantly switching between the two target categories would result in 40 runs composed of one element, whereas selecting all the occurrences of one target type before turning to the second type would result in two runs, each composed of 20 elements. If we assume equal weights between the two target categories, then selection by chance would yield an average of 21 runs composed of 1.5 elements. Research on animals has shown that run behaviour is typically random when targets are conspicuous, while animals tend to select 
Runing head: Oculomotor dynamics in visual foraging

the same target type when they are cryptic (for reviews, see Bond, 2007; Punzalan, Rodd, \& Hughes, 2005). To statistically determine whether the run behaviour of individuals was random, we used the One Sample Runs Tests separately for each trial and each individual (for examples of similar usage, see Jóhannesson et al., 2016; Kristjánsson et al., 2014). This allowed quantifying the proportion of trials that were non-random at the $p<.05$ level (adjusted using Bonferroni correction for multiple tests) for each individual and each of the four conditions (mouse-feature, mouse-conjunction, gaze-feature and gaze-conjunction). The proportion of nonrandom trials was then used to identify potential normal- and super foragers. Other behavioural dependent variables were the average number of errors (i.e., average number of distractor selections), inter-target times (the time that elapses between two successive target selections), switch costs (subtraction of the average inter-target times within runs from the average inter-target times between runs), and inter-target distances (distance in degrees of visual angle between two successive target selections).

The main oculomotor dependent variables were fixation duration and saccade amplitude, and especially the relationship between a given eye fixation duration and the amplitude of the subsequent saccade, which allow distinguishing between the ambient and focal modes of visual exploration (Unema et al., 2005). This relationship was assessed by calculating the " $K$ coefficient" proposed by Krejtz et al. (2016), which involves the subtraction of each saccade amplitude $\left(a_{i+1}\right)$ z-score to the $z$-score of its preceding $i^{\text {th }}$ fixation duration $\left(d_{i}\right)$ :

$$
K=\frac{1}{n} \sum_{n} K_{i} \quad \text { with } \quad K_{i}=\frac{d_{i}-\mu_{d}}{\sigma_{d}}-\frac{a_{i+1}-\mu_{a}}{\sigma_{a}}
$$

where $\mu_{d}$ and $\mu_{a}$ represent fixation duration and saccade amplitude means, respectively, and $\sigma_{d}$ and $\sigma_{a}$ their respective standard deviations over the total $n$ number of fixations. As such, positive $K$ coefficients reflect long fixation durations followed by small saccades, characteristic of focal visual exploration, whereas negative $K$ coefficients reflect the reverse, indicating visual exploration in an ambient mode. A $K$ coefficient close to zero is supposed to reflect exploration between the ambient and focal modes, or frequent switching between the 
Runing head: Oculomotor dynamics in visual foraging

two processing modes during the task (see Milisavljevic et al., 2019). As it is based on zscores, $K$ is expressed in standard deviations (e.g., $K=1$ means that the fixation duration is one standard deviation higher than the amplitude of the subsequent saccade).

We also measured the total number of fixations within a trial and the eye-target distance, which corresponds to the average distance in degrees of visual angle between the location of gaze and the location of the target being selected. Notably, this measure was previously used to distinguish between parallel search, involving saccade averaging with high eyetarget distances, and serial search, involving accurate saccades with low eye-target distances (Zelinsky et al., 1997).

All the dependent variables were analysed using a 2 (Target crypticity: feature foraging, conjunction foraging) by 2 (Effector type: computer mouse, eye gaze) repeated measures analysis of variance (ANOVA). Participants were furthermore divided into different subgroups according to the foraging strategy they used (i.e., random or non-random run types) in the four tested conditions, adding a between-subject factor to the analysis. Lastly, the spatiotemporal dynamics of the foraging were assessed by analysing the evolution of the obtained measures throughout the successive target selections within a trial, by adding the target index (from 1 to 40 ) to the design.

\section{Results}

Behavioural results on foraging measures

An effect of target crypticity on the number of runs $\left(F[1,23]=93.1, p<.0001, \eta_{p}^{2}=.80\right)$ confirmed previous findings (Kristjánsson et al., 2014) that feature foraging is associated with a higher number of runs (mean=18, s.d.=1.7) than conjunction foraging (mean=9, s.d.=5.4). As can be seen in Figure 2a, which presents a summary of the results obtained on foraging measures, this effect of target crypticity on the number of runs was found during both mouseand gaze-foraging, with no effect of the effector type $\left(F[1,23]=3.2, p>.08, \eta_{p}^{2}=.12\right)$ nor any 
Runing head: Oculomotor dynamics in visual foraging

interaction between the two factors $\left(F[1,23]=2.9, p>.10, \eta^{2}{ }_{p}=.11\right)$. Although these average results provide information about the overall foraging pattern, a closer look to the data reveals individual differences, especially in the conjunction foraging condition. For each participant and condition, we assessed the proportion of trials classified as nonrandom by the One Sample Run Tests. A large proportion of random trials (i.e., more than $50 \%$ of the trials) indicates a strategy of locating the nearest target and switching frequently between target categories, whereas a majority of nonrandom trials indicates a strategy of exhausting one entire target category before switching to the other category. This analysis, presented in Table 1, reveals that although there is a general tendency for random selection during feature foraging and nonrandom selection during conjunction foraging, participants can be divided into three subgroups according to the proportion of nonrandom trials. Participants s01 to s11 mainly foraged randomly during feature foraging while they showed a high proportion of nonrandom trials during conjunction foraging. In what follows, we will refer to this subgroup as "normal foragers", following the terminology proposed by Kristjánsson et al. (2014). Participants s21 to s24 mostly foraged randomly, independently of target crypticity and effector type, with less than $50 \%$ of nonrandom trials per condition. These participants seemed to correspond to the "super foragers" reported in previous studies (Jóhannesson et al., 2016, 2017; Kristjánsson et al., 2014). Interestingly, contrasting mouse and gaze foraging revealed a third foraging pattern, where observers s12 to s20 behaved as normal foragers during mouse foraging, and as super foragers during gaze foraging. This subgroup will therefore be referred to as "intermediate foragers". Note that these groups were created a posteriori, based on the results from the One Sample Run Tests presented in Table 1, so which group participants would belong to was unknown before data collection. 
Runing head: Oculomotor dynamics in visual foraging

Figures $2 b, 2 c$ and $2 d$ present the behavioural foraging measures separately for normal, intermediate and super foragers, respectively. The previously described average pattern on the number of runs (Figure 2a) actually corresponds only to the behaviour of the 11 normal foragers (Figure 2b). The three-way interaction between effector type (mouse, gaze), target crypticity (feature, conjunction) and subgroup (normal foragers, intermediate foragers, super foragers $)$ is indeed highly significant $\left(F[2,21]=42.6, p<.0005, \eta^{2}{ }_{p}=.63\right)$, and shows that although the number of runs during feature foraging does not differ between the three subgroups (all $p>.05$ at Tukey HSD post-hoc tests), it varies during conjunction foraging, so that it reflects the strategy employed by observers. During mouse-conjunction foraging, normal foragers show a smaller number of runs than intermediate and super foragers (both $p<.0005$ at Tukey HSD post-hoc tests), whereas the number of runs does not differ between intermediate and super foragers ( $p>.05$ at Tukey HSD post-hoc test). During gazeconjunction foraging however, normal and intermediate foragers showed similar run numbers $(p>.05)$ whereas super foragers showed a higher number of runs than both of the other groups (both $p$ 's<.05).

\section{Insert Figure 2 about here}

The analysis of the average number of errors (Figure 2a) showed an interaction between target crypticity and effector type, with a higher number of errors during gaze-conjunction foraging than in all other conditions $\left(F[1,23]=33.0, p<.0001, \eta^{2}{ }_{p}=.59\right)$. The number of errors for the three remaining conditions was very small and did not differ between them (all $p>.05$ at Tukey HSD post-hoc tests). When adding the foraging subgroups to the analysis (Figures $2 b$ to $2 d$ ), the interaction between effector type, target crypticity, and foraging subgroup $\left(F[2,21]=4.9, p<.01, \eta_{p}^{2}=.32\right)$ showed that during gaze-conjunction foraging, super foragers made a higher number of errors than intermediate foragers, who made more errors than 
Runing head: Oculomotor dynamics in visual foraging

normal foragers (all $p<.05$ at Tukey HSD post-hoc tests). This seems to suggest that switching between target types during conjunction foraging comes at the cost of accuracy.

Lastly, conjunction foraging led to higher inter-target times $\left(F[1.23]=153.2, p<.0001, \eta_{p}^{2}=.87\right)$ and higher inter-target distances $\left(F[1,23]=182.5, p<.0001, \eta^{2}{ }_{p}=.89\right)$ than feature foraging, and this occurred for all the three subgroups (see the two last rows in Figure 2). All three subgroups also showed higher inter-target times during gaze (mean=773 ms, s.d.=133 ms) than mouse (mean=662, s.d. $=49 \mathrm{~ms})$ foraging $\left(F[1,23]=23.4, p<.0001, \eta^{2}=.53\right)$. One could however argue that this difference in inter-target times between mouse- and gaze foraging could be driven by the $200 \mathrm{~ms}$ fixation time needed for target selection during gaze foraging. In an additional analysis, we analysed "fixation-based" inter-target times, that correspond to the time elapsed between the end of the eye fixation associated with target $i$ and the start of the eye fixation associated with target $i+1$. This additional analysis again revealed higher inter-target times during gaze (mean=359 ms, s.d.=145 ms) than mouse (mean=259 ms, s.d. $=95 \mathrm{~ms})$ foraging $\left(F[1,23]=31.8, p<.0001, \eta_{p}^{2}=.58\right)$, confirming that the effect of effector type is not driven by methodological factors. Note that participants are more likely to select the targets at the end of eye fixations, which explains why the "fixation-based" inter-target times are shorter than the traditional calculation presented in Figure 2. Conjunction foraging was moreover associated with high switch costs (mean=331 ms, s.d. $=234 \mathrm{~ms}$ ) while switch costs during feature foraging were close to zero (mean=24 ms, s.d. $=44 \mathrm{~ms} ; F[1,23]=79.9$, $\left.p<.0001, \eta^{2}=.78\right)$. Switch costs did not differ between mouse- (mean=158 ms, s.d.=125 ms) and gaze- $($ mean=197 ms, s.d. $=153 \mathrm{~ms})$ foraging $\left(F[1,23]=1.6, p>.20, \eta^{2}=.06\right)$. This means that for both effector types, during conjunction foraging the first target in a run was associated with higher inter-target times than targets selected within a run, whereas this was not the case during feature foraging. 
Runing head: Oculomotor dynamics in visual foraging

Insert Figure 3 about here

When analysing inter-target times across the successive target selections within a trial, other differences between the four foraging tasks emerged. This analysis is presented in Figure 3 (given the high range of inter-target times, we use a log-scaled y-axis in the figure to make the differences between conditions more easily observable). The first main result is that the target selected last within a trial is on average associated with higher selection times than the previously selected targets, especially in the conjunction conditions (see Figure 3a). An ANOVA run only on the data from the target selected last showed higher inter-target times for conjunction (mean=2007 ms, s.d.=581 ms) than feature (mean=875 ms, s.d.=379 ms) foraging $\left(F[1,23]=125.3, p<.0001, \eta_{p}^{2}=.84\right)$. This analysis did not reveal any effect of the effector type on the fortieth target selection $(p>.05)$. However, the same analysis run on data from the 38 other target selections (we excluded the first and last target selections) revealed, in contrast, that gaze foraging is associated with higher inter-target times (mean=752 ms, s.d.=117 ms) than mouse (mean=631 ms, s.d.=38 ms) foraging (all $p$ 's $<.001$ at Tukey HSD post-hoc tests). Interestingly, the analysis also revealed a "peak" in inter-target times at the twenty-first target selection during conjunction foraging, with higher inter-target times than at most of the other target selections in the trial (significantly different from 29 out of the 37 other target selections at $p<.05$ at the Tukey HSD post-hoc tests). This pattern seemed to reflect the transition from the first long run to the second one, as most of the observers completed these conjunction conditions by exhausting one entire target category before turning to the second one. Indeed, in Figures $3 b, 3 c$ and $3 d$ we separately examined the evolution of inter-target times across target selections for normal, intermediate and super foragers, respectively. For normal foragers, the twenty-first target selection showed significantly higher inter-target times than most of the other target selections (28 significant differences out of 37 at $p<.05$ at Tukey HSD post-hoc tests) with no difference between 
Runing head: Oculomotor dynamics in visual foraging

mouse and gaze foraging. Consistent with their foraging strategy, intermediate foragers only showed this peak during gaze foraging (significantly different from 25 out of 37 other target selections at $p<.05$ at Tukey HSD post-hoc tests). The results for super foragers are considerably noisy, probably because of the small number of participants in this subgroup, but it seems that although inter-target times were higher for gaze-conjunction foraging than the other conditions, they remained similar across target selections (see Figure $3 \mathrm{~d}$ ). Note that the discrepancy between the results at the last target selection and those at the previous target selections is particularly visible for intermediate and super foragers, who both showed higher inter-target times during mouse-conjunction than gaze-conjunction foraging at the last target selection; whereas the reverse was observed across the previous target selections (see Figures 3c and 3d).

Insert Figure 4 about here

The analysis of inter-target distances over the successive target selections is presented in Figure 4a. As for inter-target times, the analysis showed higher inter-target distances for the target selected last than for previous selections. Moreover, during conjunction foraging intertarget distances gradually increased to form a "peak" at the twenty-first selection, decreased abruptly at the twenty-second one, and then gradually increased again over the next target selections. As for inter-target times, this pattern of results seemed to mimic the foraging strategy used by normal foragers, i.e., exhausting one entire target category before turning to the second one. The separate analyses of normal, intermediate, and super foragers confirmed the link with foraging strategies, as this pattern was only found for normal foragers (Figure 4b), who showed higher inter-target distance at the twenty-first target selection than at 24 out of the 37 other target selections ( $p^{\prime} s<.05$ at Tukey HSD post-hoc tests). The intermediate foragers showed this pattern during gaze foraging only (with 23 out of the 37 
Runing head: Oculomotor dynamics in visual foraging

comparisons being significant at $p<.05$ at Tukey HSD post-hoc tests; see Figure 4c), and the super foragers did not show any peaks in inter-target distance at the twenty-first target selection (all $p$ 's $>.05$, see Figure $4 d$ ). Notably, even though this last subgroup included only four observers, the data were less variable than for inter-target times, suggesting that intertarget distance could be a more suitable measure than inter-target times for assessing the evolution of the foraging strategy used by observers over the successive target selections.

\section{Eye movement dynamics during foraging}

Figure 5a presents the average results for eye movement dynamics during the foraging tasks. Conjunction foraging was associated with a higher number of eye fixations $\left(F[1,23]=349.7, p<.0001, \eta_{p}^{2}=.94\right)$, lower fixation duration $\left(F[1,23]=128.8, p<.0001, \eta_{p}^{2}=.85\right)$ and higher saccade amplitude $\left(F[1,23]=87.7, p<.0001, \eta^{2}=.79\right)$ than feature foraging. These are all indicators of visual exploration in an ambient mode during conjunction foraging compared to a focal mode during feature foraging (see Tatler \& Vincent, 2008; Unema et al., 2005). Figures $5 b$ to $5 d$ show that these results were similar for all observers, irrespective of the foraging strategy used. The interaction between effector type and target crypticity on fixation duration $\left(F[1,23]=22.8, p<.0001, \eta_{p}^{2}=.52\right)$ moreover showed that during conjunction foraging, foraging with eye gaze led to higher fixation durations (mean=330 ms, s.d. $=56 \mathrm{~ms}$ ) than foraging with the computer mouse (mean=295 ms, s.d.=36 ms). This was not the case during feature foraging ( $p>.05$ at Tukey HSD post-hoc test). This effect of effector type was however only found for the intermediate foragers (Tukey HSD post-hoc tests, see Figure 5).

\section{Insert Figure 5 about here}

Based on these results, we calculated the $K$ coefficient (Krejtz et al., 2016; see the data analysis section for more details) that represents the relationship between each fixation 
Runing head: Oculomotor dynamics in visual foraging

duration and the amplitude of the subsequent saccade. This analysis is presented in Figure $6 a$. The $K$ coefficients on the average data from all the 24 observers were different from zero in all four conditions (all p's<.05 at one-sample Student $t$ tests), and the ANOVA revealed an effect of target crypticity $\left(F[1,23]=189.8, p<.0001, \eta^{2}=.89\right), \mathrm{K}$ being positive during feature foraging (mouse: $1.57 \pm 1.15$, gaze: $1.26 \pm 1.84$ ) and negative during conjunction foraging (mouse: $-1.12 \pm 1.78$, gaze: $-.71 \pm 2.28$ ). The $K$ coefficients did not differ between mouse- and gaze foraging $(F<1)$. As can be seen in Figure $6 a$, the separate analysis of data from the three foraging subgroups revealed that the exploration remained in a focal mode (positive $K$ ) during feature foraging for all observers; while during conjunction foraging, normal foragers explored the visual scene in an ambient mode (negative $K$ ), whereas super foragers showed $K$ coefficients close to zero, indicating visual exploration in between the ambient and focal modes, or frequent switches between ambient and focal modes during the task. Intermediate foragers showed intermediate results, behaving as normal foragers do during mouse foraging and as super foragers do during gaze foraging.

Insert Figure 6 about here

Note however that fixation duration and saccade amplitude were here computed for all the eye movements made during the task. As can be seen in the first row of Figure 5, participants sometimes made many eye fixations, especially during the conjunction foraging tasks. Only 40 of these eye fixations however correspond to target selections. When restricting the analysis of the $K$ coefficient to the 40 "critical" fixations and their subsequent saccades, there were interestingly even larger differences between subgroups. This analysis, presented in Figure 6b, shows that during conjunction foraging, super foragers turn to a focal processing mode (positive $K$ coefficient) right after each target selection. During mouse foraging, super foragers are also the only subgroup changing their foraging strategy between 
Runing head: Oculomotor dynamics in visual foraging

the 40 fixations corresponding to target selection (Figure $6 \mathrm{~b}$ ) and other eye fixations within a trial (Figure 6a). Note that these results on critical fixations are found irrespective of the landing position of the subsequent saccades (i.e., the differences in fixation duration and saccade amplitude between normal, intermediate and super foragers are found for both the critical fixations followed by saccades landing on another target and for the critical fixations followed by saccades landing on a distractor or an empty area). Conversely, Figure 6c presents the $\mathrm{K}$ coefficient only for the fixations that are not associated with target selection (i.e., "non-critical" fixations). Overall, this analysis replicates the results obtained for the entire dataset (Figure 6a). Feature and conjunction foraging are associated with the focal and ambient modes of visual exploration, respectively, but when comparing normal, intermediate and super foragers we see that switching between target categories is associated more with a focal visual exploration mode.

Finally, the analysis of the distance between target location and eye fixation location associated with target selection (last row in Figure 5) showed higher eye-target distance during mouse- than gaze foraging $\left(F[1,23]=77.4, p<.0001, \eta_{p}^{2}=.77\right)$. This result is however not surprising as during gaze foraging participants are forced to accurately fixate the targets to select them. More interestingly, when foraging with the computer mouse, conjunction foraging was associated with higher eye-target distance than feature foraging $(F[1,23]=20.9$, $p<.0005, \eta_{p}^{2}=.48$ ). As shown in Figure 5 , this seems to be the case for all observers, whatever their foraging strategy. As for inter-target times and distances in Figures 3 and 4, we examined how eye fixation duration, saccade amplitude and eye-target distance evolved over the successive target selections within a trial. The measures were however consistent from the second to the thirty-ninth target selection, suggesting that observers did not change their oculomotor behaviour throughout the successive target selections within a foraging trial. 
Runing head: Oculomotor dynamics in visual foraging

\section{Discussion}

Our study confirmed previous findings of Kristjánsson et al. (2014) that like animal foragers, humans adapt their strategy to target crypticity. During conspicuous feature foraging, they select targets in numerous short runs, whereas during cryptic conjunction foraging, they select targets in much fewer and longer runs. But one novel finding here is that this behaviour was observed during both mouse- and gaze-foraging, suggesting that the effector type used to perform the task does not influence the overall foraging strategy. This result is in contrast with previous findings of Jóhannesson et al. (2016) who observed more numerous short runs when observers foraged with eye gaze than with their fingers. Remember, however, that this previous study involved fewer visual stimuli during gaze foraging than during finger foraging, and that the observed difference could be due to the difference in task difficulty induced by the differing number of targets and distractors. As the targets were fewer on the screen, they were, most likely, more conspicuous than in the finger-foraging task, both during feature and conjunction foraging. In our study, gaze and mouse foraging involved the same number of stimuli and were visually identical, allowing full comparison between tasks. The analysis of the individual profiles in foraging strategy actually suggested that gaze foraging is a more difficult task than mouse foraging. Indeed, although some "super foragers" were identified during mouse foraging, such performance was much rarer during gaze foraging. Critically, the comparison between feature and conjunction foraging during mouse and gaze foraging revealed a third group of individuals, that we call "intermediate foragers", behaving like super foragers during mouse foraging (i.e., switching frequently between the two target categories irrespective of target crypticity) but acting as normal foragers during gaze foraging (i.e., changing their foraging strategy depending on target crypticity). Obviously, these individuals were able to switch between two cryptic target categories during mouse-conjunction foraging, but they chose not to do so when foraging with eye gaze. Moreover, they made more errors during that condition, reinforcing the idea that gazeconjunction foraging was a more difficult task than mouse-conjunction foraging. Gaze 
Runing head: Oculomotor dynamics in visual foraging

foraging was also associated with higher inter-target times than mouse foraging. Importantly, additional analyses on "fixation-based" inter-target times (i.e., the difference between the ending time of the eye fixation associated with target $i$ and the starting time of the eye fixation associated with target $i+1$ ) show that this difference is not driven by the fixation time needed for target selection during gaze foraging. Moreover, eye fixation duration was only higher during gaze foraging than mouse foraging for intermediate foragers, and only in the conjunction condition. It is therefore likely that this difference in fixation duration between effector types was linked to the change in foraging strategy made by intermediate foragers, rather than to methodological factors such as the fixation time needed for target selection during gaze foraging.

\section{Are super-foragers superb?}

Notably, our results suggest that the "super foragers" may not be as "superb" as has been thought. Super- and intermediate foragers made numerous errors, especially during gazeconjunction foraging, while normal foragers made very few errors in all conditions. The optimal strategy for efficient foraging might therefore be to not switch between cryptic target types and to adapt the foraging strategy to target crypticity. Note that we used the term "super foragers" with reference to terminology that has been used in previous studies (e.g., Clarke, Irons, James, Leber, \& Hunt, 2018; Jóhannesson et al., 2016, 2017; Kristjánsson et al., 2014), but in light of our findings, a more appropriate term could be "suboptimal foragers". One could indeed argue that the four super - or suboptimal - foragers, who never changed their foraging strategy between tasks, were actually persevering with the same behaviour, maybe reflecting weak attentional flexibility. This would moreover be consistent with studies of children that have shown that foraging behaviour correlates with attentional flexibility capacity (Ólafsdóttir et al., 2016, 2019). Jóhannesson et al. (2017) did not find correlations between foraging behaviour and cognitive abilities in adults, but their study did not involve any measurement of attentional flexibility. Their measures only involved working memory and 
Runing head: Oculomotor dynamics in visual foraging

inhibition capacities, that did not correlate well with foraging strategies. Moreover, Jóhannesson et al. (2017)'s study only involved a finger foraging task, and the intermediate foragers could therefore not be distinguished from the super/suboptimal foragers. Intermediate foragers might have higher attentional flexibility than super/suboptimal foragers, as they adapted their strategy to target crypticity during gaze foraging, and having them in the same group as super/suboptimal foragers might have weakened the observed correlations between finger-foraging behaviour and cognitive ability capacities. Further studies are needed to investigate the links between foraging behaviour and attentional flexibility in adults, by varying target crypticity and constrasting different effector types.

\section{Within-trial performance}

Apart from the number of runs and the number of errors, the other traditional behavioural foraging measures did not vary very much by foraging strategy. When we analysed the evolution of these measures over successive target selections within a trial, however, differences emerged between the three subgroups of participants. Individuals proceeding in two long runs during conjunction foraging (normal foragers and intermediate foragers when foraging with eye gaze) showed peaks in inter-target times and distances at the twenty-first target selection, reflecting the transition from the first run to the second; whereas individuals foraging randomly (super foragers and intermediate foragers during mouse-foraging) showed constant inter-target times and distances over the successive target selections. Hence, the evolution of these measures within trials accurately captures the foraging strategy being used. Note that these analyses could not be performed with standard single-target visual search tasks, as trials would only include one target selection. These analyses clearly highlight the usefulness of foraging tasks compared to traditional visual search tasks, that do not enable such analyses over time, as there is only one target. Moreover, these analyses showed that the target selected last was associated with much higher inter-target time and distance than the previous ones. Recent research (Kristjánsson, Thornton, Chetverikov, \& 
Runing head: Oculomotor dynamics in visual foraging

Kristjánsson, 2020) has revealed that the well-known set-size effects during feature- and conjunction search are actually only found for the last target selection of foraging tasks, suggesting that traditional single-target visual search experiments only reflect the last target selection of foraging behaviours. In single-target visual search, researchers may only have access to this last target selection, that captures only a very specific aspect of search behaviour, that is probably not very representative of the overall search dynamics in natural environments.

\section{Oculomotor measures}

We also assessed oculomotor dynamics during foraging tasks. Oculomotor dynamics did not vary much from the second to the thirty-ninth target selection, conjunction foraging being associated with lower fixation duration and higher saccade amplitude than feature foraging. This suggests that foraging through cryptic targets is associated with ambient visual exploration, whereas foraging through conspicuous targets is associated with focal visual exploration. This is surprising, as ambient exploration has been proposed to be linked to bottom-up processes, and focal exploration to top-down processes (Unema et al., 2005). We expected ambient exploration to be involved during conspicuous/feature foraging, not during cryptic/conjunction foraging. But the study of Unema et al. (2005) was based on visual explorations of natural visual scenes and did not involve any visual search or foraging. The mechanisms involved might therefore have been different. During feature foraging, observers mainly located the nearest target and progressed gradually through the visual scene. It therefore makes sense that their visual exploration would be organized in a focal mode, as they only made small jumps from one target to another. In contrast, during conjunction foraging most observers exhausted one entire target category before turning to the other. As such, while they proceeded through the visual scene, the remaining targets from the category being selected became more and more sparse, and participants had to make larger saccades to reach the remaining targets. This behaviour is therefore reflected in ambient 
Runing head: Oculomotor dynamics in visual foraging

visual exploration. But in our study, ambient exploration does not seem to be associated with bottom-up processing. In contrast, it seems that observers' strategy during conjunction foraging is goal-driven, especially when participants are still searching for the sparse remaining targets from the category selected first while plenty of targets from the second category are available in the display. In conclusion, our study shows that ambient exploration is not always associated with bottom-up processes, and that the involvement of high-level processes depends more on the task and strategy used by observers than on the mode in which they explore the visual scene. Overall, this may suggest that the ambient/focal distinction may need some revision (see also Milisavljevic et al., 2019).

Finally, the analysis of oculomotor dynamics revealed that during mouse foraging, the eyetarget distance was higher during conjunction- than during feature foraging. In that case, higher inter-target distance might be related to saccade averaging (Zelinsky et al., 1997). Our paradigm did indeed involve the presentation of many stimuli at the same time, and proximal stimuli are known to influence the saccade landing position, that is directed to the "centre of gravity" of the visual scene instead of landing on the exact target position (Coren \& Hoenig, 1972; Findlay, 1982). During sequences of saccades, this phenomenon has been proposed to reflect parallel processing of differing visual stimulations (Zelinsky et al., 1997). In the current study, this would mean that mouse-conjunction foraging was associated with more parallel processing than mouse-feature foraging. This is in accordance with the observation that the ambient mode of visual exploration was used during conjunction foraging. Note however that a higher inter-target distance could also be due to higher anticipation of the next target, the eyes being allowed to move before the target has been selected with the mouse. This could also explain why gaze foraging was more difficult than mouse foraging. Indeed, during gaze foraging observers had to accurately fixate the target to select it. They therefore could not anticipate the next target, which may have increased task difficulty compared to mouse foraging, where participants were free to move their eyes whenever they wanted. 
Runing head: Oculomotor dynamics in visual foraging

\section{Foraging strategies}

Our suggestion that the ambient/focal distinction may require modification is consistent with the separate analysis of the visual exploration mode for the three different subgroups (Figure 6). During conjunction foraging, the normal foragers stayed in an ambient mode throughout the trial, while super (or suboptimal) foragers seemed to employ visual exploration in between the ambient and focal modes, or to frequently switch between the two. Note that the ambient/focal distinction is here assumed to be a continuum on which performance can vary according to time, tasks and individuals. This switching might actually reflect attentional fluctuations, where the super/suboptimal foragers briefly changed to more focal exploration (here assumed to be related to bottom-up processes) before turning back to a more ambient mode (here assumed to be related to top-down processes) allowing the successful completion of the task. These supposed attentional fluctuations are moreover consistent with the higher number of distractor selections observed for super/suboptimal foragers than the other participants.

Interestingly, Boot, Becic, \& Kramer (2009) showed that when the task is not too demanding, observers usually tend to prefer a given "default" strategy, even if it is not the most relevant one; but that when the task becomes too demanding, participants modify their strategy and adopt one that is more adaptive to the task. Hence, intermediate foragers may by default favour the "super-foraging" strategy, i.e., locate the nearest target and gradually proceed in the visual field. But during gaze-conjunction foraging, the task may have become too demanding, making them change their strategy to a "normal" one, more adaptive to the task. The analysis of the visual exploration mode actually showed that the super/suboptimal foragers also seemed to modify their strategy during gaze-conjunction foraging. After each target selection, they turned to more focal visual exploration, allowing them to locate the nearest target (see Figure 6b). They therefore seemed to have noticed that their "default" strategy was maladaptive to the task, but they unfortunately turned to another maladaptive 
Runing head: Oculomotor dynamics in visual foraging

strategy, leading to numerous errors. Note that although these participants modified their visual exploration mode, they did not change their overall behaviour. In contrast, they changed their visual exploration mode so as to keep their "by default" behavioural foraging strategy in all tasks.

\section{Conclusions}

The analysis of oculomotor dynamics during both mouse and gaze foraging provides new insights into the optimal behaviour for efficient human foraging. We showed that what has been called "normal foraging" may be an efficient way of exploring complex environments, especially when the task is demanding. We moreover have shown that the foraging strategies used by observers are associated with different oculomotor dynamics. Individuals explore the visual scene in a focal mode when foraging through conspicuous targets, whereas they use more ambient visual exploration for foraging through cryptic targets. Our results might therefore lead to the updating of the theories of visual attention and visual exploration that have been based on results from traditional single-target visual search tasks (see e.g., Kristjánsson, Ólafsdóttir, \& Kristjánsson, 2019). Critically, we showed that these traditional tasks only consider the last target selection in a trial, but do not reflect overall search behaviour (see also Kristjánsson et al., 2020). Multi-target foraging tasks appears, overall, to be a very efficient way of measuring the dynamics of attentional and oculomotor behaviours.

\section{References}

Bargary, G., Bosten, J. M., Goodbourn, P. T., Lawrance-Owen, A. J., Hogg, R. E., \& Mollon, J. D. (2017). Individual differences in human eye movements: An oculomotor signature? Vision Research, 141, 157-169. https://doi.org/10.1016/j.visres.2017.03.001 
Runing head: Oculomotor dynamics in visual foraging

Becker, W., \& Jürgens, R. (1979). An analysis of the saccadic system by means of double step stimuli. Vision Research, 19(9), 967-983. https://doi.org/10.1016/0042$6989(79) 90222-0$

Bond, A. B. (2007). The evolution of color polymorphism: crypticity, searching images, and apostatic selection. Annual Review of Ecology, Evolution, and Systematics, 38, 489-514. https://doi.org/10.1146/annurev.ecolsys.38.091206.095728

Boot, W. R., Becic, E., \& Kramer, A. F. (2009). Stable individual differences in search strategy?: The effect of task demands and motivational factors on scanning strategy in visual search. Journal of Vision, 9(3):7, 1-16. https://doi.org/10.1167/9.3.7

Cain, M. S., \& Mitroff, S. R. (2013). Memory for found targets interferes with subsequent performance in multiple-target visual search. Journal of Experimental Psychology: Human Perception and Performance, 39(5), 1398-1408. https://doi.org/10.1037/a0030726

Clarke, A. D. F., Dr, Irons, J., James, W., Leber, A. B., \& Hunt, A. R. (2018, November 30). Stable individual differences in strategies within, but not between, visual search tasks. https://doi.org/10.31234/osf.io/bqa5v

Coren, S., \& Hoenig, P. (1972). Effect of non-target stimuli upon length of voluntary saccades. Perceptual and motor skills, 34(2), 499-508.

Dawkins, M. (1971). Shifts of "attention" in chicks during feeding. Animal Behaviour, 19, 575582. https://doi.org/10.1016/S0003-3472(71)80114-8

Dukas, R. (2002). Behavioural and ecological consequences of limited attention. Philosophical Transactions of the Royal Society of London B: Biological Sciences, 357(1427), 1539-1547. https://doi.org/10.1098/rstb.2002.1063

Dukas, R., \& Ellner, S. (1993). Information Processing and Prey Detection. Ecology, 74(5), 1337-1346. https://doi.org/10.2307/1940064

Durand, A. C., \& Gould, G. M. (1910). A method of determining ocular dominance. Journal of the American Medical Association, 55(5), 369-370. https://doi.org/10.1001/jama.1910.04330050007004 
Runing head: Oculomotor dynamics in visual foraging

Eckstein, M. P. (2011). Visual search: A retrospective. Journal of Vision, 11(5):14, 1-36. https://doi.org/10.1167/11.5.14

Findlay, J. M. (1982). Global visual processing for saccadic eye movements. Vision Research, 22(8), 1033-1045. https://doi.org/10.1016/0042-6989(82)90040-2

Findlay, J. M., \& Walker, R. (1999). A model of saccade generation based on parallel processing and competitive inhibition. Behavioral and Brain Sciences, 22(04), 661-674.

Foulsham, T., Frost, E., \& Sage, L. (2018). Stable individual differences predict eye movements to the left, but not handedness or line bisection. Vision Research, 144, 38-46. https://doi.org/10.1016/j.visres.2018.02.002

Goldberg, J. H., \& Kotval, X. P. (1999). Computer interface evaluation using eye movements: methods and constructs. International Journal of Industrial Ergonomics, 24(6), 631-645. https://doi.org/10.1016/S0169-8141(98)00068-7

Groner, R., Walder, F., \& Groner, M. (1984). Looking at Faces: Local and Global Aspects of Scanpaths. In A. G. Gale \& F. Johnson (Ed.), Advances in Psychology (p. 523-533). https://doi.org/10.1016/S0166-4115(08)61874-9

He, P., \& Kowler, E. (1989). The role of location probability in the programming of saccades: Implications for "center-of-gravity" tendencies. Vision Research, 29(9), 1165-1181.

Hills, T. T., Kalff, C., \& Wiener, J. M. (2013). Adaptive Lévy Processes and Area-Restricted Search in Human Foraging. PloS One, 8(4):e60488, 1-7. https://doi.org/10.1371/journal.pone.0060488

Jóhannesson, Ó. I., Kristjánsson, Á., \& Thornton, I. M. (2017). Are Foraging Patterns in Humans Related to Working Memory and Inhibitory Control? Japanese Psychological Research, 59(2), 152-166. https://doi.org/10.1111/jpr.12152

Jóhannesson, Ó. I., Thornton, I. M., Smith, I. J., Chetverikov, A., \& Kristjánsson, Á. (2016). Visual Foraging With Fingers and Eye Gaze. i-Perception, 7(2), 1-18. https://doi.org/10.1177/2041669516637279 
Runing head: Oculomotor dynamics in visual foraging

Krejtz, K., Duchowski, A., Krejtz, I., Szarkowska, A., \& Kopacz, A. (2016). Discerning Ambient/Focal Attention with Coefficient K. ACM Trans. Appl. Percept., 13(3):11, 1-20. https://doi.org/10.1145/2896452

Kristjánsson, Á. (2015). Reconsidering Visual Search. i-Perception, 6(6), 1-14. https://doi.org/10.1177/2041669515614670

Kristjánsson, Á., Jóhannesson, Ó. I., \& Thornton, I. M. (2014). Common Attentional Constraints in Visual Foraging. PloS One, 9(6):e100752, 1-9. https://doi.org/10.1371/journal.pone.0100752

Kristjánsson, Á., Ólafsdóttir, I. M., \& Kristjánsson, T. (2019). Visual foraging tasks provide new insights into the orienting of visual attention: methodological considerations. In S. Pollmann (Ed.), Neuromethods. Humana Press. (p. 1-19).

Kristjánsson, T., \& Kristjánsson, Á. (2018). Foraging through multiple target categories reveals the flexibility of visual working memory. Acta Psychologica, 183, 108-115. https://doi.org/10.1016/j.actpsy.2017.12.005

Kristjánsson, T., Thornton, I.M., Chetverikov, A. \& Kristjánsson, Á. (2020). Dynamics of visual attention revealed in foraging tasks. Cognition, 194:104032, 1-13. https://doi.org/10.1016/j.cognition.2019.104032

Kristjánsson, T., Thornton, I. M., \& Kristjánsson, Á. (2018). Time limits during visual foraging reveal flexible working memory templates. J Exp Psychol Hum Percept Perform, 44(6), $827-835$

Liversedge, S. P., \& Findlay, J. M. (2000). Saccadic eye movements and cognition. Trends in Cognitive Sciences, 4(1), 6-14.

McLaughlin, S. C. (1967). Parametric adjustment in saccadic eye movements. Perception \& Psychophysics, 2(8), 359-362.

Milisavljevic, A., Le Bras, T., Mancas, M., Petermann, C., Gosselin, B., \& Doré-Mazars, K. (2019). Towards a better description of visual exploration through temporal dynamic of ambient and focal modes. Proceedings of the 11th ACM Symposium on Eye Tracking \& Applications:66, 1-4. https://doi.org/10.1145/3314111.3323075 
Runing head: Oculomotor dynamics in visual foraging

Moran, R., Zehetleitner, M., Liesefeld, H. R., Müller, H. J., \& Usher, M. (2016). Serial vs. parallel models of attention in visual search: accounting for benchmark RT-distributions. Psychonomic Bulletin \& Review, 23(5), 1300-1315. https://doi.org/10.3758/s13423-0150978-1

Noton, D., \& Stark, L. (1971). Scanpaths in saccadic eye movements while viewing and recognizing patterns. Vision Research, 11(9), 929-942. https://doi.org/10.1016/00426989(71)90213-6

Ólafsdóttir, I. M., Gestsdóttir, S., \& Kristjánsson, Á. (2019). Visual foraging and executive functions: A developmental perspective. Acta Psychologica, 193, 203-213. https://doi.org/10.1016/j.actpsy.2019.01.005

Ólafsdóttir, I. M., Kristjánsson, T., Gestsdóttir, S., Jóhannesson, Ó. I., \& Kristjánsson, Á. (2016). Understanding visual attention in childhood: Insights from a new visual foraging task. Cognitive Research: Principles and Implications, 1:18, 1-12. https://doi.org/10.1186/s41235-016-0016-5

Oldfield, R. C. (1971). The assessment and analysis of handedness: The Edinburgh inventory. Neuropsychologia, 9(1), 97-113. https://doi.org/10.1016/0028-3932(71)90067-4

Over, E. A. B., Hooge, I. T. C., Vlaskamp, B. N. S., \& Erkelens, C. J. (2007). Coarse-to-fine eye movement strategy in visual search. Vision Research, 47(17), 2272-2280. https://doi.org/10.1016/j.visres.2007.05.002

Punzalan, D., Rodd, F. H., \& Hughes, K. A. (2005). Perceptual processes and the maintenance of polymorphism through frequency-dependent predation. Evolutionary Ecology, 19(3), 303-320. https://doi.org/10.1007/s10682-005-2777-z

Rayner, K. (2009) Eye movements and attention in reading, scene perception, and visual search. The Quarterly Journal of Experimental Psychology, 62(8), 1457-1506. https://doi.org/10.1080/17470210902816461

Tagu, J., Doré-Mazars, K., Vergne, J., Lemoine-Lardennois, C., \& Vergilino-Perez, D. (2018a). Quantifying eye dominance strength - New insights into the neurophysiological 
Runing head: Oculomotor dynamics in visual foraging

bases of saccadic asymmetries. Neuropsychologia, 117, 530-540.

https://doi.org/10.1016/j.neuropsychologia.2018.07.020

Tagu, J., Doré-Mazars, K., Vergne, J., Lemoine-Lardennois, C., \& Vergilino-Perez, D. (2018b). Recentering bias for temporal saccades only: Evidence from binocular recordings of eye movements. Journal of Vision, 18(1):10, 1-16.

https://doi.org/10.1167/18.1.10

Tatler, B. W., \& Vincent, B. T. (2008). Systematic tendencies in scene viewing. Journal of Eye Movement Research, 2(2):5, 1-18. https://doi.org/10.16910/jemr.2.2.5

Tinbergen, L. (1960). The natural control of insects in pinewoods. Archives Néerlandaises de Zoologie, 13(3), 265-343. https://doi.org/10.1163/036551660X00053

Treisman, A. M., \& Gelade, G. (1980). A feature-integration theory of attention. Cognitive Psychology, 12(1), 97-136. https://doi.org/10.1016/0010-0285(80)90005-5

Unema, P. J. A., Pannasch, S., Joos, M., \& Velichkovsky, B. M. (2005). Time course of information processing during scene perception: The relationship between saccade amplitude and fixation duration. Visual Cognition, 12(3), 473-494.

https://doi.org/10.1080/13506280444000409

Velichkovsky, B. M., Rothert, A., Kopf, M., Dornhöfer, S. M., \& Joos, M. (2002). Towards an express-diagnostics for level of processing and hazard perception. Transportation Research Part F: Traffic Psychology and Behaviour, 5(2), 145-156. https://doi.org/10.1016/S1369-8478(02)00013-X

Wolfe, J. M. (2013). When is it time to move to the next raspberry bush? Foraging rules in human visual search. Journal of Vision, 13(3):10, 1-17. https://doi.org/10.1167/13.3.10

Wolfe, J. M., \& Horowitz, T. S. (2017). Five factors that guide attention in visual search. Nature Human Behaviour, 1(3):0058, 1-8. https://doi.org/10.1038/s41562-017-0058

Zangemeister, W. H., Sherman, K., \& Stark, L. (1995). Evidence for a global scanpath strategy in viewing abstract compared with realistic images. Neuropsychologia, 33(8), 1009-1025. https://doi.org/10.1016/0028-3932(95)00014-T 
Runing head: Oculomotor dynamics in visual foraging

Zelinsky, G. J., Rao, R. P. N., Hayhoe, M. M., \& Ballard, D. H. (1997). Eye movements reveal the spatiotemporal dynamics of visual search. Psychological Science, 8(6), 448-453.

https://doi.org/10.1111/j.1467-9280.1997.tb00459.x 
Runing head: Oculomotor dynamics in visual foraging

\section{Figure and Table captions}

\section{Caption for Figure 1}

Figure 1. Foraging tasks. Panel (a) shows the feature foraging condition, where observers had to select the red and green dots while ignoring the blue and yellow ones, or vice versa. Panel (b) shows the conjunction foraging condition, where observers had to select the red squares and green dots while ignoring the green squares and red dots, or vice versa. Participants performed these conditions both using a computer mouse and their eye gaze to select the targets.

\section{Caption for Figure 2}

Figure 2. Summary of the results obtained on behavioural foraging measures. Error bars represent the $95 \%$ confidence intervals. (a) data from all the 24 observers, (b) data from the 11 normal foragers, (c) data from the 9 intermediate foragers, (d) data from the 4 super foragers.

\section{Caption for Figure 3}

Figure 3. Time course of inter-target times within foraging trials (in milliseconds, log-scaled). Error bars represent standard errors of the mean. (a) data from all the 24 observers, (b) data from the 11 normal foragers, (c) data from the 9 intermediate foragers, (d) data from the 4 super foragers.

\section{Caption for Figure 4}

Figure 4. Time course of inter-target distance within foraging trials (in degrees of visual angle, log-scaled). Error bars represent the standard errors of the mean. (a) data from all the 
Runing head: Oculomotor dynamics in visual foraging

24 observers, (b) data from the 11 normal foragers, (c) data from the 9 intermediate foragers, (d) data from the 4 super foragers.

\section{Caption for Figure 5}

Figure 5. Summary of the results obtained on oculomotor measures. Error bars represent the $95 \%$ confidence intervals. (a) data from all the 24 observers, (b) data from the 11 normal foragers, (c) data from the 9 intermediate foragers, (d) data from the 4 super foragers.

\section{Caption for Figure 6}

Figure 6. $\mathrm{K}$ coefficients reflecting the visual exploration mode used by observers. (a) represents the $\mathrm{K}$ coefficients for all the eye fixations and saccades that have been executed during the tasks, whereas (b) and (c) respectively show the $\mathrm{K}$ coefficients only for the 40 eye fixations associated with target selections and the ones that are not associated with target selections. In all panels, the first column shows average data for all the 24 observers whereas the three other columns show the same analysis separately for each subgroup. The error bars represent the standard errors of the mean.

\section{Caption for Table 1}

Table 1. Proportion of trials classified as nonrandom by the One Sample Runs Tests as a function of participant and condition. Participants s01 to s11 are classified as normal foragers, participants s12 to s20 as intermediate foragers, and participants s21 to s24 as super foragers. 


\section{a. feature foraging}

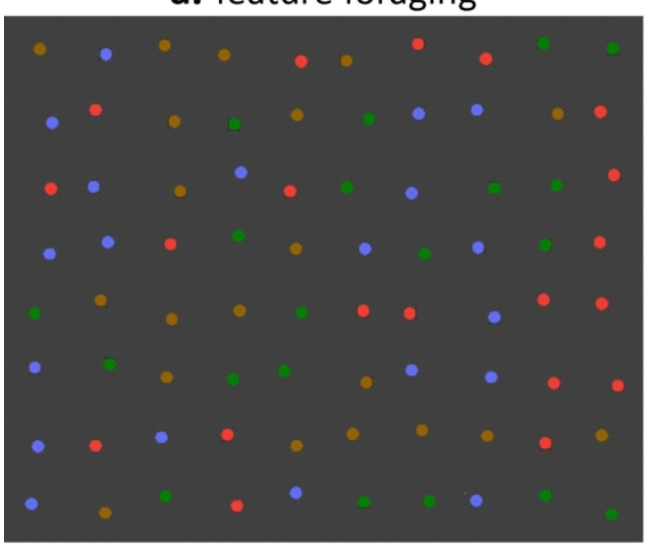

b. conjunction foraging

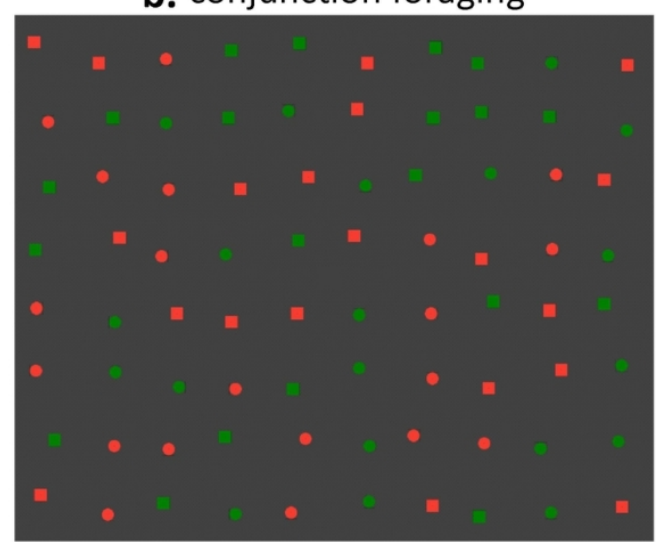

Figure 1. Foraging tasks. Panel (a) shows the feature foraging condition, where observers had to select the red and green dots while ignoring the blue and yellow ones, or vice versa. Panel (b) shows the conjunction foraging condition, where observers had to select the red squares and green dots while ignoring the green squares and red dots, or vice versa. Participants performed these conditions both using a computer mouse and their eye gaze to select the targets.

$125 \times 54 \mathrm{~mm}(300 \times 300$ DPI $)$ 
a. average results
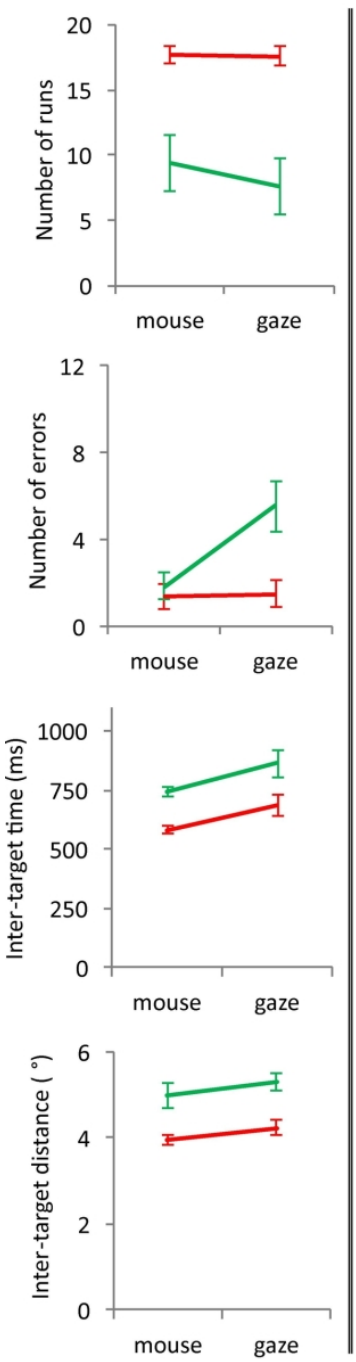

b. normal foragers
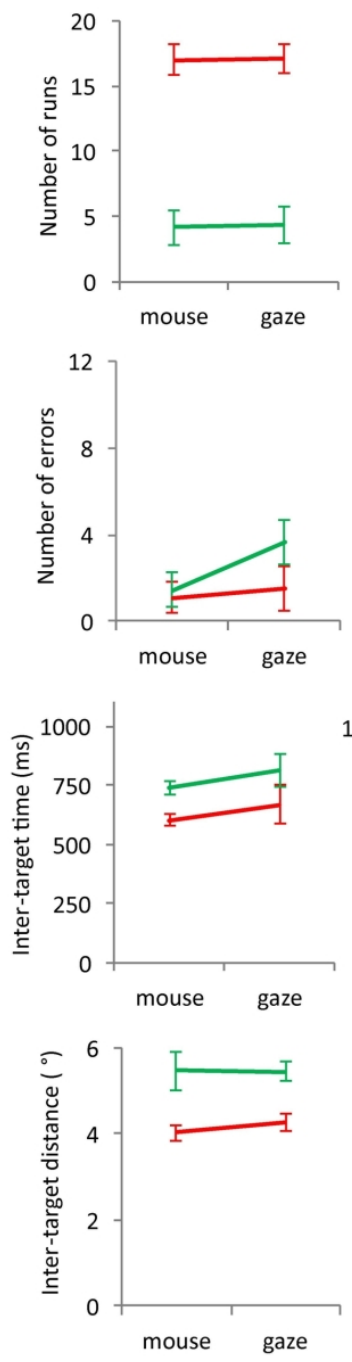

c. intermediate foragers

d. super foragers
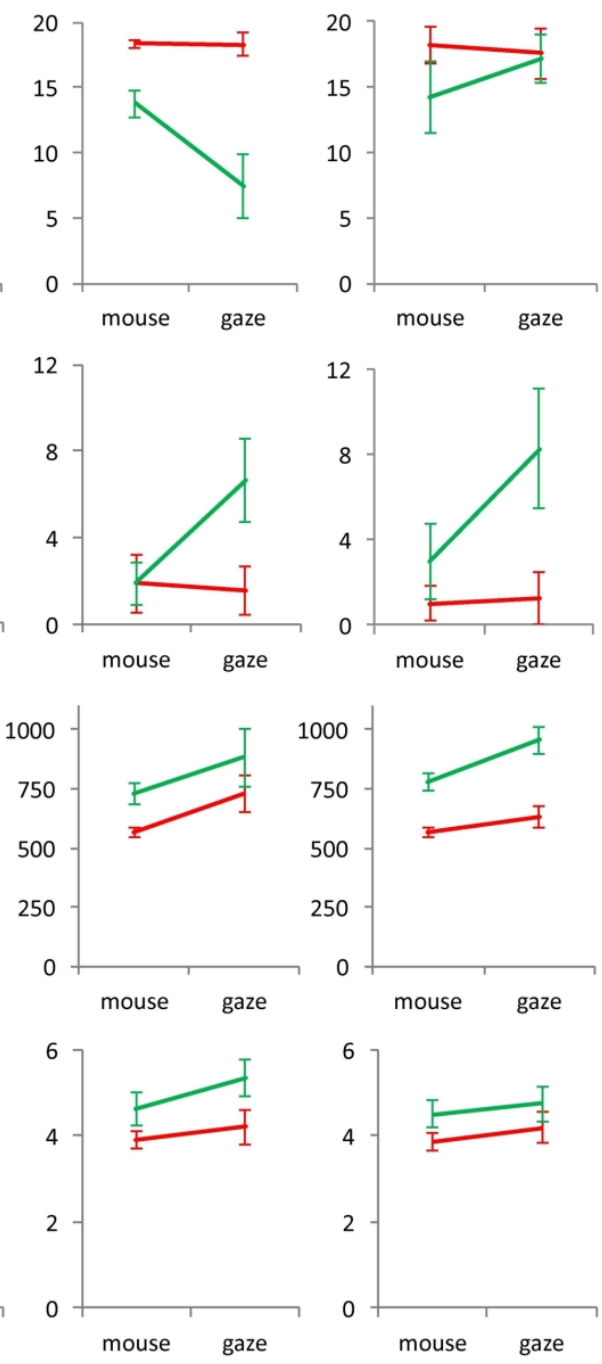

Figure 2. Summary of the results obtained on behavioural foraging measures. Error bars represent the $95 \%$ confidence intervals. (a) data from all the 24 observers, (b) data from the 11 normal foragers, (c) data from the 9 intermediate foragers, (d) data from the 4 super foragers.

$184 \times 207 \mathrm{~mm}(300 \times 300$ DPI $)$ 
a. average results
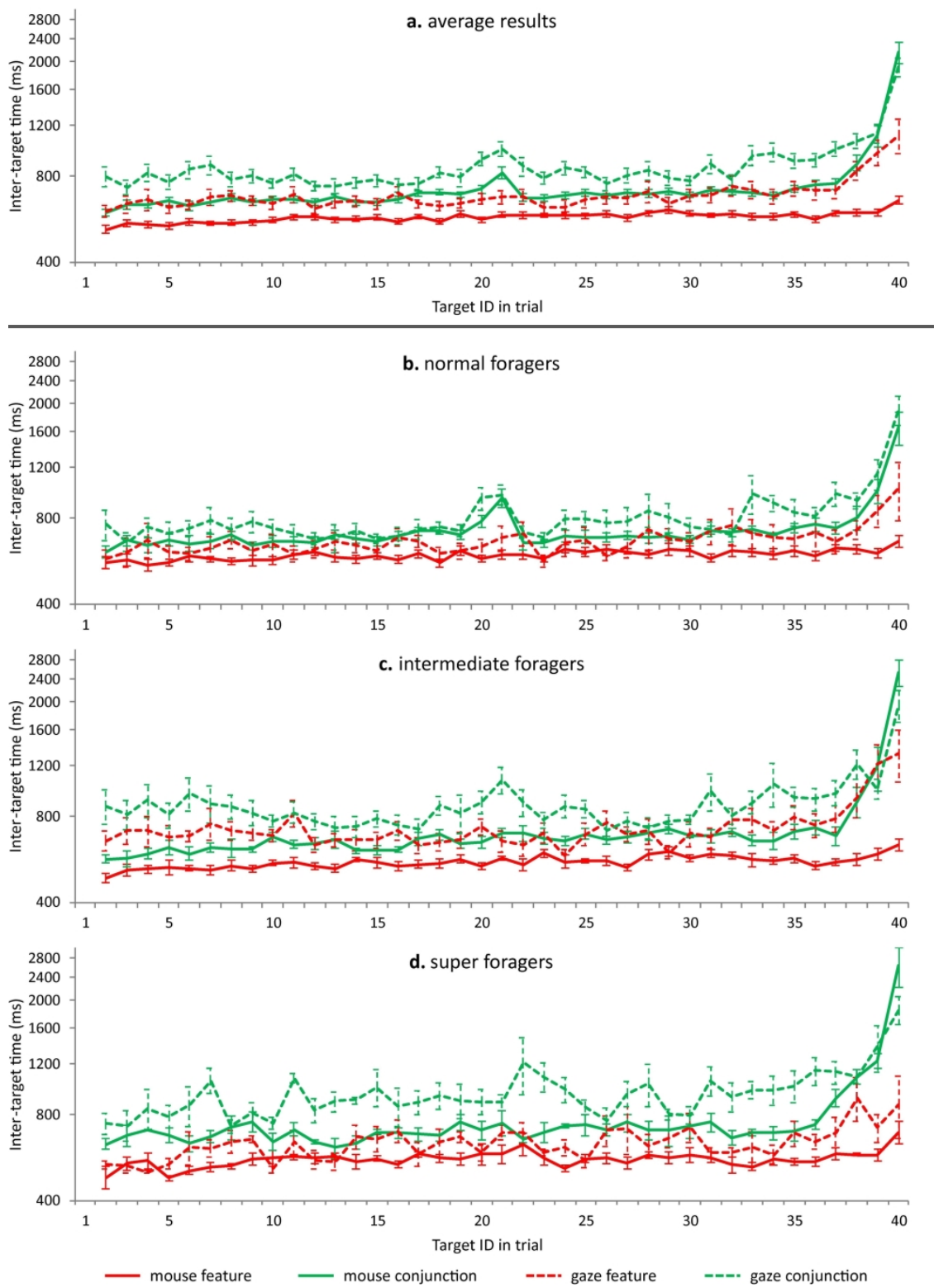

Figure 3. Time course of inter-target times within foraging trials (in milliseconds, log-scaled). Error bars represent standard errors of the mean. (a) data from all the 24 observers, (b) data from the 11 normal foragers, (c) data from the 9 intermediate foragers, (d) data from the 4 super foragers.

$191 \times 267 \mathrm{~mm}(300 \times 300 \mathrm{DPI})$ 

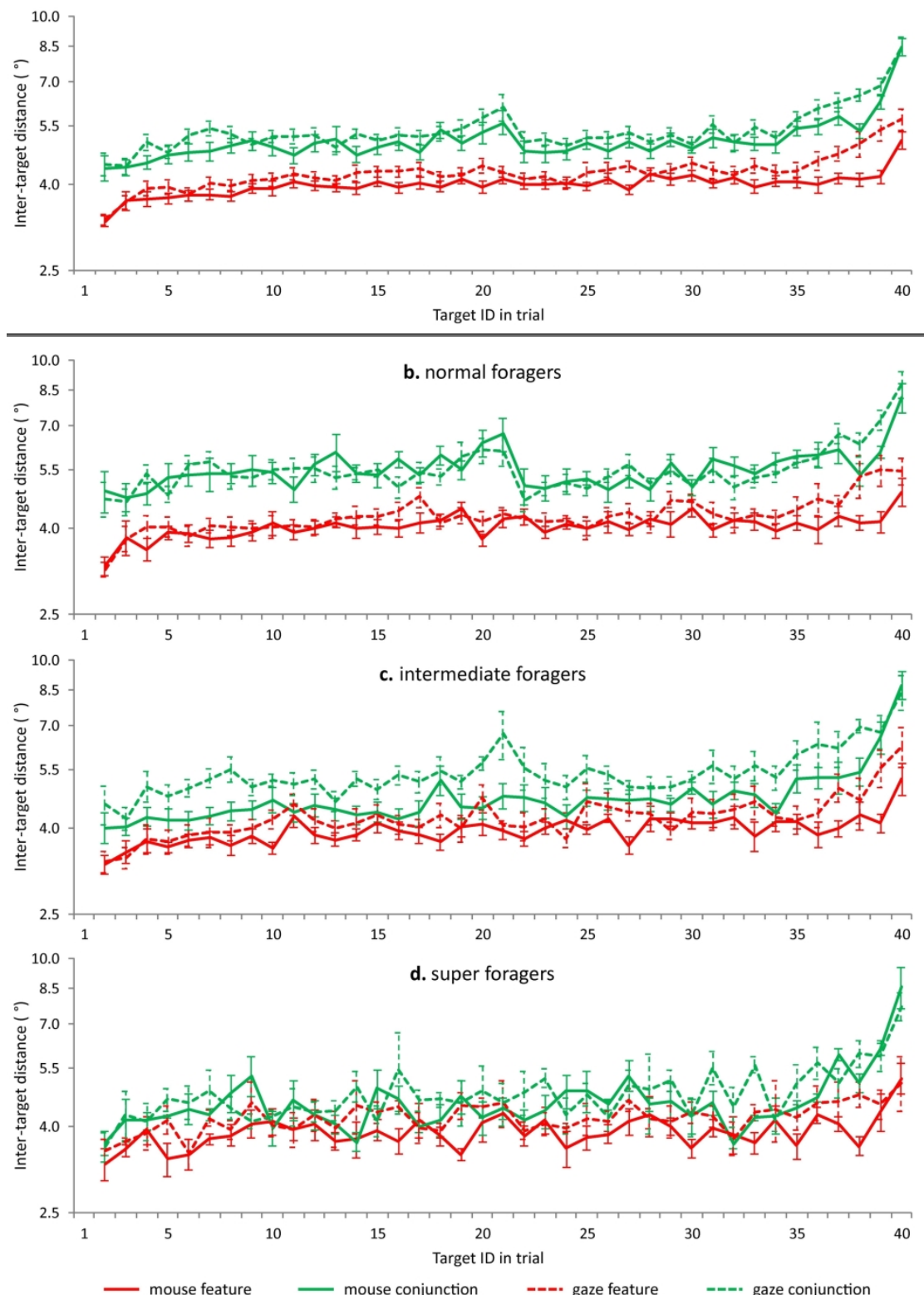

Figure 4. Time course of inter-target distance within foraging trials (in degrees of visual angle, log-scaled). Error bars represent the standard errors of the mean. (a) data from all the 24 observers, (b) data from the 11 normal foragers, (c) data from the 9 intermediate foragers, (d) data from the 4 super foragers.

$191 \times 267 \mathrm{~mm}(300 \times 300 \mathrm{DPI})$ 
a. average results
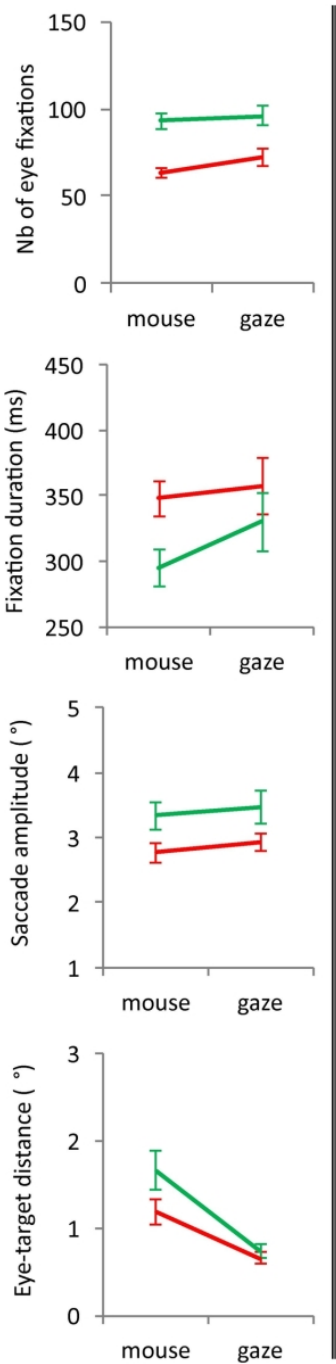

b. normal foragers

c. intermediate foragers

d. super foragers
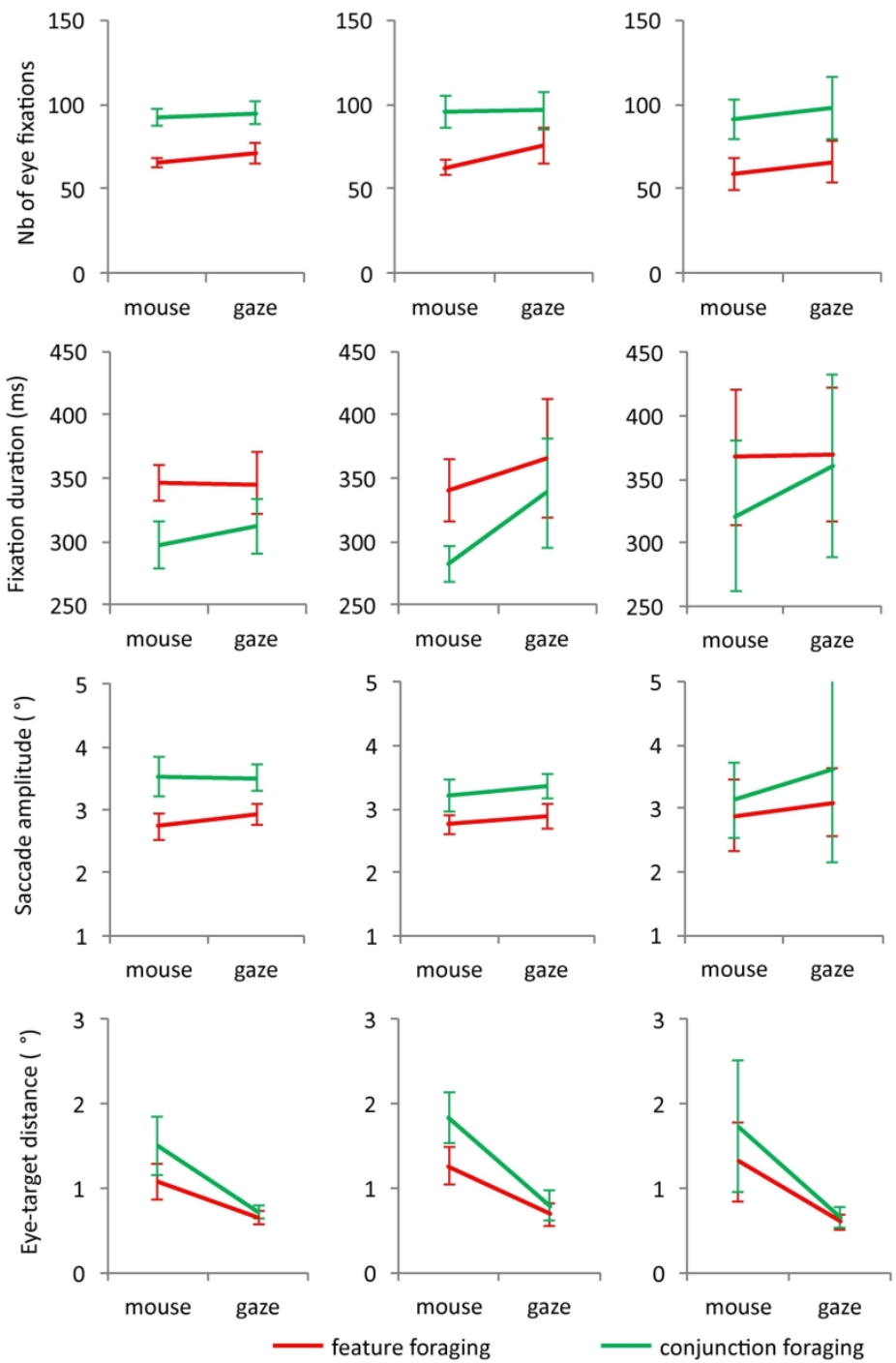

Figure 5. Summary of the results obtained on oculomotor measures. Error bars represent the $95 \%$ confidence intervals. (a) data from all the 24 observers, (b) data from the 11 normal foragers, (c) data from the 9 intermediate foragers, (d) data from the 4 super foragers.

$183 \times 204 \mathrm{~mm}(300 \times 300$ DPI $)$ 

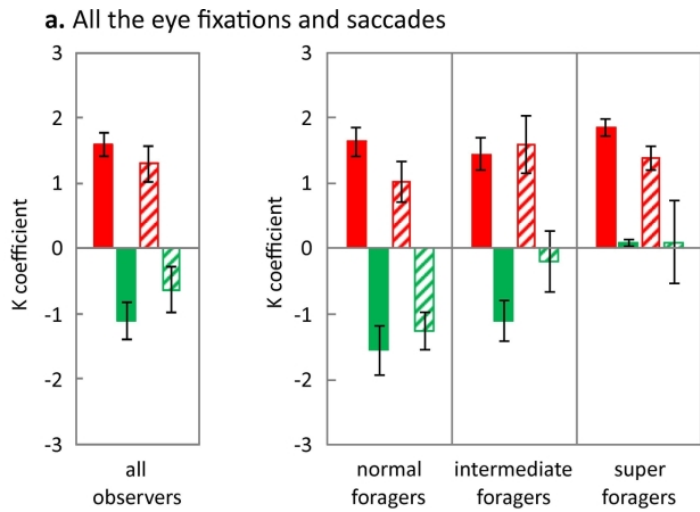

- mouse feature

mouse conjunction

$\square$ gaze feature

c. Fixations not associated with target selection
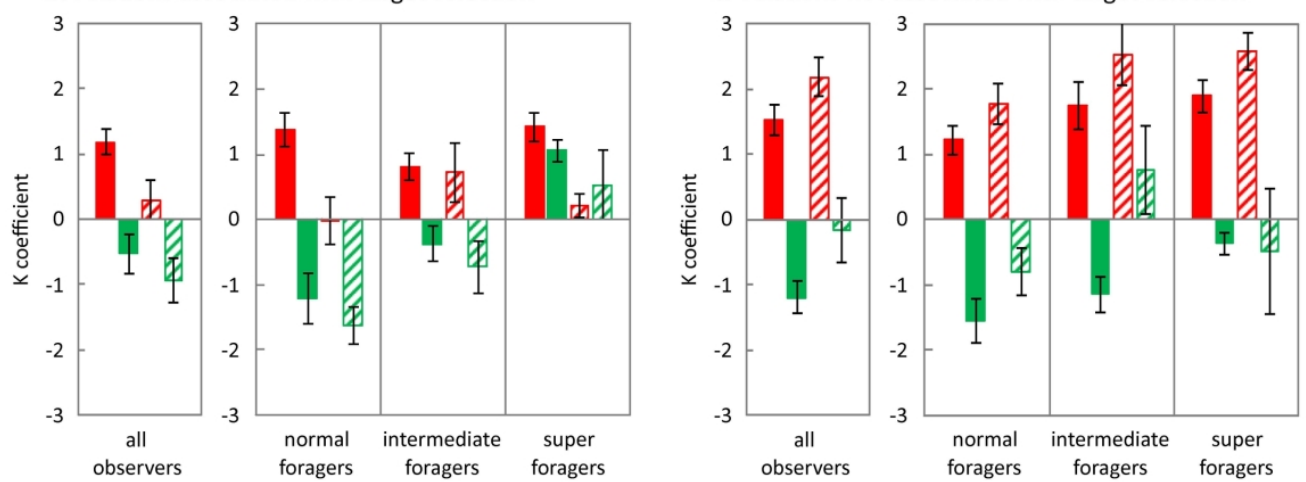

Figure $6 . \mathrm{K}$ coefficients reflecting the visual exploration mode used by observers. (a) represents the $\mathrm{K}$ coefficients for all the eye fixations and saccades that have been executed during the tasks, whereas (b) and (c) respectively show the $\mathrm{K}$ coefficients only for the 40 eye fixations associated with target selections and the ones that are not associated with target selections. In all panels, the first column shows average data for all the 24 observers whereas the three other columns show the same analysis separately for each subgroup. The error bars represent the standard errors of the mean.

$206 \times 167 \mathrm{~mm}(300 \times 300$ DPI $)$ 
Table 1. Proportion of trials classified as nonrandom by the One Sample Runs Tests as a function of participant and condition. Participants s01 to s 11 are classified as normal foragers, participants s 12 to s 20 as intermediate foragers, and participants s21 to s24 as super foragers.

\begin{tabular}{|c|c|c|c|c|}
\hline \multirow[b]{2}{*}{ Participant ID } & \multicolumn{2}{|c|}{ Mouse foraging } & \multicolumn{2}{|c|}{ Gaze foraging } \\
\hline & Feature & Conjunction & Feature & Conjunction \\
\hline s01 & $19 \%$ & $100 \%$ & $0 \%$ & $100 \%$ \\
\hline s02 & $50 \%$ & $100 \%$ & $0 \%$ & $88 \%$ \\
\hline s03 & $0 \%$ & $75 \%$ & $13 \%$ & $81 \%$ \\
\hline s04 & $6 \%$ & $81 \%$ & $0 \%$ & $81 \%$ \\
\hline s05 & $13 \%$ & $100 \%$ & $0 \%$ & $100 \%$ \\
\hline s06 & $0 \%$ & $100 \%$ & $13 \%$ & $100 \%$ \\
\hline s07 & $0 \%$ & $94 \%$ & $0 \%$ & $100 \%$ \\
\hline s08 & $0 \%$ & $81 \%$ & $0 \%$ & $81 \%$ \\
\hline s09 & $0 \%$ & $100 \%$ & $0 \%$ & $100 \%$ \\
\hline s10 & $0 \%$ & $94 \%$ & $13 \%$ & $100 \%$ \\
\hline s11 & $0 \%$ & $81 \%$ & $31 \%$ & $100 \%$ \\
\hline s12 & $0 \%$ & $6 \%$ & $0 \%$ & $60 \%$ \\
\hline s13 & $0 \%$ & $38 \%$ & $6 \%$ & $56 \%$ \\
\hline s14 & $0 \%$ & $0 \%$ & $0 \%$ & $94 \%$ \\
\hline s15 & $6 \%$ & $31 \%$ & $0 \%$ & $69 \%$ \\
\hline s16 & $0 \%$ & $6 \%$ & $0 \%$ & $100 \%$ \\
\hline s17 & $6 \%$ & $44 \%$ & $0 \%$ & $100 \%$ \\
\hline s18 & $0 \%$ & $38 \%$ & $0 \%$ & $88 \%$ \\
\hline s19 & $0 \%$ & $13 \%$ & $13 \%$ & $50 \%$ \\
\hline s20 & $6 \%$ & $19 \%$ & $13 \%$ & $69 \%$ \\
\hline s21 & $0 \%$ & $0 \%$ & $0 \%$ & $0 \%$ \\
\hline s22 & $0 \%$ & $13 \%$ & $13 \%$ & $13 \%$ \\
\hline s23 & $6 \%$ & $44 \%$ & $0 \%$ & $19 \%$ \\
\hline s24 & $0 \%$ & $19 \%$ & $0 \%$ & $0 \%$ \\
\hline Average & $5 \%$ & $53 \%$ & $5 \%$ & $73 \%$ \\
\hline SD & $11 \%$ & $38 \%$ & $8 \%$ & $34 \%$ \\
\hline
\end{tabular}

